\title{
RECURSOS FISIOTERAPÊUTICOS UTILIZADOS NO TRATAMENTO DE PATOLOGIAS MAIS COMUNS EM IDOSOS \\ - um estudo de revisão
}

\author{
Fernando Campbell Bordiak ${ }^{a}$ \\ Izabele Fagundes Machado ${ }^{b}$ \\ Gabriel Machado da Silva Alves ${ }^{b}$ \\ Jacyara Peruzzi ${ }^{b}$ \\ Líria Tainá Manhães ${ }^{b}$ \\ Raquel Aguiar Franco ${ }^{b}$
}

Fontes de auxílio: Vice-reitoria de pesquisa da UNESA.

\begin{abstract}
Resumo
Objetivo: Apresentar quais são os principais recursos terapêuticos aplicados na reabilitação do idoso de acordo com suas patologias mais comuns. Metodologia: Foram selecionados artigos científicos nas bases de pesquisa Pubmed, Medline, Bireme, e Scielo, seguindo indicadores específicos. Resultados: Foram selecionados 17 artigos científicos em língua portuguesa e inglesa. Para alicerçar o estudo foram inclusos 3 livros na referência. Principais achados: $O$ acidente vascular encefálico (AVE), a doença de Parkinson e a esclerose múltipla são os principais transtornos neurológicos que acometem os idosos. Os transtornos ortopédicos comuns em idosos são a artrite reumatoide e a osteoartrite. A participação dos idosos em atividades físicas regulares minimiza o declínio funcional. Cinesioterapia e hidrocinesioterapia resultam em melhoria funcional articular. Alongamentos musculares são recomendados para idosos para aumentar a flexibilidade. A Eletrotermofototerapia pode ser útil no tratamento de patologias do aparelho locomotor de idosos. Conclusão: Os casos patológicos comuns em idosos são AVE, doença de Parkinson, esclerose múltipla, artrite reumatoide, e osteoartrites. As formas mais frequentes de intervenção fisioterapêutica podem ocorrer com a realização de cinesioterapia, alongamentos musculares, hidrocinesioterapia, e recursos de eletrotermofototerapia.
\end{abstract}

Palavras-chave: Fisioterapia; Idoso; Tratamento.

Correspondence Author: Fernando Campbell Bordiak (Campbell, FB) - drcampbell@ig.com.br

a. Professor da disciplina de Eletrotermofototerapia - UNESA Campus Norte Shopping.

b. Graduandos em Fisioterapia - UNESA Campus Norte Shopping. 


\title{
PHYSICAL THERAPY RESOURCES USED IN THE TREATMENT OF MOST COMMON DISEASES IN ELDERY
}

\author{
- a review study
}

\begin{abstract}
Objective: Present the main therapeutical resources applied at eldery rehabilitation, according to their most common diseases. Methodology: Scientific articles were selected, based at Pubmed, Medline, Bireme and Scielo, following specific indicators. Results: 17 scientific articles were selected in English and Portuguese idioms. In order to strength this study, 3 books were included as reference. Main findings: Stroke, Parkinson's disease, and multiple sclerosis are the commonly neurological disease among eldery. Rheumatoid arthritis and osteoarthritis are the most common orthopaedical diseases among eldery. The participation of eldery in regular physical activities decreases the functional limitation. Kinesiotherapy and hydrokinesiotherapy results in articular motion improvement. Muscle stretching are recommended for eldery because of the flexibility empowerment. Electrothermalphototherapy can be helpful in the treatment of the eldery musculoskeletal diseases. Conclusion: The common diseases in eldery are stroke, Parkinson's disease, multiple sclerosis, rheumatoid arthritis and osteoarthritis. The most frequently forms of physical therapy interventions can occur performing Kinesiotherapy, muscle stretching, hydrokinesiotherapy, and electrothermalphototherapy resources.
\end{abstract}

Keywords: Physical therapy; Eldery, treatment.

\section{INTRODUÇÃO}

O processo natural de envelhecimento é responsável por diversas modificações e alterações orgânicas e funcionais que ocorrem de forma progressiva, caracterizando-se por diminuição da massa e da força muscular, diminuição da atividade do sistema nervoso, alterações cardiovasculares, diminuição do equilíbrio, diminuição da capacidade pulmonar, fraqueza e diminuição da massa óssea e comprometimento no funcionamento de diversos órgãos e sistemas. ${ }^{(1,2)}$

No idoso, os transtornos ortopédicos mais comuns são as artroses, a artrite reumatoide, e a osteoporose, que pode predispor à ocorrência de fraturas. ${ }^{(1)}$ Já os principais transtornos neurológicos são o acidente vascular encefálico (AVE), a doença de Parkinson e a esclerose múltipla. Os transtornos neurológicos em idosos causam importantes reflexos de limitações funcionais, que serão tratados através de diferentes técnicas fisioterapêuticas. ${ }^{(3,4)}$

A cinesioterapia atua como um ótimo tratamento para idosos por ser uma terapia através do movimento. Uma estratégia para minimizar o declínio funcional é a participação dos idosos em atividades físicas regulares, com programas de exercícios direcionados para ganho de força, melhora do equilíbrio, melhora da amplitude articular entre outros benefícios. Por se tratar da realização de movimentos físicos planejados, a cinesioterapia para idosos tem a característica de poder ser direcionada para atendimentos individuais ou em grupo. ${ }^{(5)}$ 
A eletrotermofototerapia é constituída por recursos que resultam em ação analgésica e antiinflamatória. ${ }^{(6)}$ Estudos foram conclusivos que o laser de baixa intensidade e o TENS são eficazes na melhora da dor em pacientes com osteoartrose e na meIhora da funcionalidade desses pacientes, e mais, esses recursos físicos, ao contrário dos fármacos analgésicos e anti-inflamatórios, não apresentam os indesejáveis efeitos adversos. . $^{(7,8)}$

A incontinência urinária (IU) não é problema exclusivamente do processo de envelhecimento, porém com o avanço da idade, se agrava consideravelmente, seja por comprometimentos de outras patologias ou por efeito de medicamentos utilizados para tratar tais distúrbios ou disfunções orgânicas. ${ }^{(9)}$

Tendo em vista a variedade de opções disponíveis para utilização pelo fisioterapeuta no tratamento de diferentes acometimentos em idosos, este estudo tem como objetivo apresentar quais são os principais recursos terapêuticos aplicados na reabilitação do idoso de acordo com as disfunções e patologias mais comuns.

\section{METODOLOGIA}

\section{MECANISMO DE BUSCA}

Para elaboração deste estudo, foram selecionados artigos científicos relacionados à temática de idosos e patologias associadas nas seguintes bases de pesquisa nacionais e internacionais: Pubmed, Medline, Bireme, Scielo e Google. Os indicadores utilizados no processo de busca foram: Idosos, eldery, artrose. fratura, artrite reumatoide, rheumatoid arthritis, osteoporose, osteoporosis, AVC, doença de Parkinson, parkinson's disease, esclerose múltipla, multiple sclerosis e incontinência urinária, urinary incontinence.

Para identificação de intervenções fisioterapêuticas, os termos acima foram combinados com as palavras fisioterapia e physical therapy. Após o levantamento quantitativo de artigos científicos, foi realizada análise qualitativa de acordo com os objetivos deste estudo, mediante leitura. O levantamento de artigos científicos foi realizado no período entre março e junho de 2013.

\section{CRITÉRIOS DE EXCLUSÃO}

Foram considerados como critérios de exclusão, artigos de revisão que não abordavam intervenções terapêuticas sobre idosos, assim como textos de caráter não-científico sobre o assunto.

\section{RESULTADOS}

Foram selecionados 17 artigos científicos, sendo 13 em língua portuguesa e 4 em língua inglesa, seguindo os propósitos expostos na metodologia. Para alicerçar o estudo com algumas informações substanciais, foram ainda incluídos 3 livros sobre gerontologia, neurologia e condicionamento físico.

\section{PRINCIPAIS ACHADOS}

O AVE, a doença de Parkinson e a esclerose múltipla são os principais transtornos neurológicos que acometem os idosos. O AVE geralmente é de início súbito, e acarreta em déficits motores e/ou sensitivos, que refletem o local e a extensão do comprometimento cerebral. A doença de Parkinson que é uma doença degenerativa do sistema nervoso central, que acomete os núcleos da base, na substância negra e no corpo estriado, onde se encontra praticamente toda dopamina do cérebro humano. (3) A esclerose múltipla, compromete a substância branca hemisférica, causa déficits neuropsicológicos na função executiva, memória de trabalho e velocidade de processamento de informação. (4)

No idoso, ocorre redução da massa óssea, mais frequente em mulheres.(1) Os transtornos ortopédicos comuns em idosos são a artrite reumatoide e a osteoartrite. A artrite reumatoide é uma doença sistêmica auto-imune, cuja principal característica é a sinovite crônica, simétrica e erosiva das articulações periféricas. ${ }^{(10)} \mathrm{A}$ osteoartrite, é um processo induzido nas articulações por influências mecânicas, 
metabólicas e genéticas, causando perda de cartilagem e hipertrofia óssea. ${ }^{(6)}$

Uma estratégia para minimizar o declínio funcional é a participação dos idosos em atividades físicas regulares, com programas de exercícios direcionados para ganho de força, melhora do equilíbrio, melhora da amplitude articular entre outros benefícios. ${ }^{(5)}$ Por se tratar da realização de movimentos físicos planejados, a cinesioterapia direcionada para idosos tem a predominância de exercícios de alongamentos, exercícios resistidos e atividades de relaxamento, auxiliando na prevenção e no retardo dos efeitos do envelhecimento. ${ }^{(11)}$

A cinesioterapia atua como um ótimo tratamento para idosos por ser uma terapia através do movimento, que resulta em melhoria funcional articular. $\mathrm{Na}$ terceira idade, os declínios funcionais do organismo tornam-se mais evidentes. ${ }^{(5)}$ Ocorrem disfunções musculoesqueléticas, diminuição da agilidade, da coordenação, do equilíbrio, da flexibilidade e da mobilidade articular, que geram menor qualidade na contração muscular, déficit na coordenação dos movimentos e maior probabilidade do indivíduo de sofrer quedas. ${ }^{(11)}$

Dentre os diversos tipos de atividade física, exercícios de alongamento são recomendados para idosos como meio de manter ou aumentar os níveis de flexibilidade, uma vez que permitem uma fácil execução e são relativamente seguros.(12) A flexibilidade, caracterizada pela máxima amplitude de movimento de uma ou mais articulações, é um dos principais componentes de capacidade funcional, sendo necessária para realização de atividades da vida diária. ${ }^{(13)}$ Estudos têm demonstrado que exercícios de alongamento podem proporcionar aumento na amplitude articular. ${ }^{(14)}$

Os alongamentos devem ser realizados durante o aquecimento e ao final da sessão. A melhoria da amplitude articular aumenta o desempenho muscular, reduz o risco de lesão e melhora a nutrição da cartilagem. Articulações dolorosas não devem ser alongadas de forma excessiva a ponto de causar dor, com todos os movimentos devendo ser feitos de modo a conseguir a máxima amplitude articular, de forma assintomática. A aplicação de hipertermoterapia antes do alongamento reduz a dor e aumenta a amplitude articular. ${ }^{(15)}$

Os exercícios terapêuticos em hidrocinesioterapia têm papel importante na prevenção, manutenção e melhora da funcionalidade do idoso. A água oferece suporte e minimiza o estresse biomecânico nos músculos e articulações. Outros benefícios associados com os exercícios aquáticos em água quente, são a melhora da circulação sanguínea, aumento da força muscular, aumento da amplitude articular, relaxamento muscular, diminuição temporária do nível de dor, melhora da confiança e da capacidade funcional. ${ }^{(16)}$

As propriedades físicas da água irão auxiliar os idosos, resultando em benefícios de aumento de amplitude articular, flexibilidade, atenuação do impacto articular, aumento de força e resistência, incremento na ação dos sistemas cardiovascular e respiratório, relaxamento de tecidos moles, e na eliminação das tensões mentais, entre outros. ${ }^{\left({ }^{(7)}\right)}$

Técnicas de eletrotermofototerapia podem ser úteis no tratamento de diferentes disfunções do aparelho locomotor de idosos. Morgan et al (2011), relatam ser possível a diminuição de dor articular em portadores de osteoartrite de joelho com o uso do TENS, ${ }^{(8)}$ sendo também proveitoso o tratamento com laser sobre os mesmos casos, com sinais de melhora a curto prazo. ${ }^{(7)}$

A eletroestimulação neuromuscular pode ser proveitosa no tratamento de patologias degenerativas, de joelho e coluna vertebral. A ação seletiva da eletroestimulação sobre os músculos multífidos lombares, após a realização de exercícios de estabilização, pode atuar na recuperação do seu tônus e trofismo, promovendo uma melhor estabilização da coluna vertebral, resultando em menos dor pelo paciente. ${ }^{(18)}$ Resultados positivos da eletroestimulação também podem ser percebidos se aplicada sobre o quadríceps, pois seu incremento na ação muscular, pode colaborar positivamente sobre a estabilização articular do joelho. ${ }^{(19)}$

Sendo uma modalidade terapêutica conservadora, a fisioterapia tem sido bastante importante 
na melhora do estado geral dos pacientes, podendo inclusive, promover a cura em alguns casos. Situações muito específicas fazem parte de suas possibilidades de ação, como por exemplo, sobre a IU, que é uma disfunção que pode ser ocasionada após AVE ou cirurgias pélvicas. Técnicas de cinesioterapia e eletroestimulação neuromuscular podem se tornar positivas sobre esses casos, reduzindo e até abolindo a perda urinária. $(9,20)$

\section{CONCLUSÃO}

Tendo em vista os resultados apresentados, podemos concluir que a Fisioterapia pode apresentar importante eficácia na abordagem terapêutica sobre casos patológicos comuns em idosos, que são AVE, doença de Parkinson, esclerose múltipla, artrite reumatoide, e osteoartrites. É também relevante seu papel sobre a disfunção de IU. Suas formas de intervenção podem ocorrer com a realização de um programa de exercícios, alongamentos musculares, hidrocinesioterapia, e recursos de eletrotermofototerapia

\section{REFERÊNCIAS}

1. Nobrega AC, et al. Posicionamento oficial da Sociedade Brasileira de Medicina do Esporte e da Sociedade Brasileira de Geriatria e Gerontologia: atividade física e saúde no idoso; Rev Bras Med Esporte 1999 Nov-Dez;5(6):207-11.

2. Najas, MS. Gerontologia. São Paulo: Atheneu;1996.

3. Stokes, MC. Neurologia para fisioterapeutas. $2^{a}$ ed. São Paulo: Premier;2000.

4. Ferreira FO, et al. Velocidade de processamento, sintomas depressivos e memória de trabalho: comparação entre idosos e portadores de esclerose múltipla. Psicol Reflex Crit. 2011 Abr/Jun;24(2):367-80.

5. Santos LT, et al. Efeitos da cinesioterapia sobre os níveis de IGF-1, força muscular e autonomia funcional em mulheres idosas. Rev Bras
Cineantropom Desempenho Hum. 2010 Nov/ Dez;12(6):451-6.

6. Biasoli, MC. Tratamento fisioterápico na terceira idade. Rev Bras Med. 2007 Nov; 64(Ed. Esp.):62-8.

7. Fukuda VO, et al. Eficácia a curto prazo do laser de baixa intensidade em pacientes com osteoartrite do joelho: Ensaio clínico aleatório, placebo controlado e duplo-cego; Rev Bras Ortop. 2011 Set/Out; 46(5):526-33.

8. Morgan CR, et al. Estudo da estimulação elétrica nervosa transcutânea nível sensório para efeito de analgesia em pacientes com osteoartrose de joelho. Fisioter Mov. 2011 Out/Dez; 24(4):637-46.

9. Guedes JM, et al. Incontinência urinária no idoso: Abordagem fisioterapêutica. Rev Bras Ciênc Envelhecimento Humano 2006 Jan/Jul;3(1):105-113.

10. Brandão L, et al. Avaliação da qualidade de vida na artrite reumatoide: Revisão atualizada. Rev Bras Reumatol. 1997 Set/ Out;37(5):275-81.

11. Constantini A, et al. Exercícios físicos e fatores de quedas em idosos. Revista Polidisciplinar Eletrônica da Faculdade Guairacá. 2011 Dez;3(2):17-30.

12. Gobbi S, Villar R, Zago AS. Bases teóricopráticas do condicionamento físico. Rio de Janeiro: Guanabara Koogan;2005.

13. American College of Sports Medicine, Chodzko-Zajko WJ, Proctor DN, Singh $M A$, et al. Exercise and physical activity for older adults: Position stand from the American College of Sports Medicine and the American Heart Association. Med Sci Sports Exerc. 2009, Jul;41(7):1510-30.

14. Gallon D, et al. The effects of stretching on the flexibility, muscle performance and functionality of institutionalized older women. Braz J Med Biol Res. 2011 Mar. 44(3):229-35.

15. Pedrinelli $A$, et al. $\bigcirc$ efeito da atividade física no aparelho locomotor do idoso. Rev Bras Ortop. 2009 Mar/Abr;44(2):96-101. 
16. Gabilan YP, et al. Fisioterapia Aquática para Reabilitação Vestibular. Acta ORL. 2006;24(1):25-30.

17. Assis, RS, et al. A hidroginástica melhora o condicionamento físico em idosos. Rev Bras de Prescrição e Fisiologia do Exercício 2007 Set/Out;1(5):62-75.

18. Bordiak FC, Silva EB. Eletroestimulação e core training sobre dor e arco de movimento na lombalgia. Fisioter Mov. 2012 Out-Dez;25(4):759-66.
19. Gaines JM, Metter EJ, Talbot LA. The effect of neuromuscular electrical stimulation on arthritis knee pain in older adults with osteoarthritis of the knee. Appl Nurs Res. 2004 Ago;17(3):201-6.

20.Arvonen T, Fianu-Jonasson A, Tyni-Lenné R. Effectiveness of two conservative modes of physical therapy in women with urinary stress incontinence.

Neurourology and Urodynamics 2001 Out/Nov;2O(5):591-9. 